\title{
Experimental research of free space optical communication based on cat's eye modulating retro-reflector using GaAs/AlGaAs multiple quantum well
}

\author{
Hua-Yan Sun, Lai-Xian Zhang ${ }^{*}$, Jian-Ying Ren and Cong-Miao Shan \\ Department of Optical-Electronic, Academy of Equipment, Beijing, 101416, China \\ E-mail: zhanglaixian@126.com \\ *Corresponding author
}

\begin{abstract}
Cat's eye modulating retro-reflector makes fast ATP, very high speed, small, light and low power consumption free space optical communication link possible. This paper analyzes the principle of free space optical communication based on cat's eye modulating retro-reflector using a multiple quantum well spatial light modulator, and introduces the principle of $\mathrm{GaAs} / \mathrm{AlGaAs}$ multiple quantum well using as a spatial light modulator. An experiment has been done to test characteristics of a GaAs/AlGaAs multiple quantum well device used as a spatial light modulator. An experimental free space optical communication system based on cat's eye modulating retro-reflector using GaAs/AlGaAs multiple quantum well was built, and a communication link was tested using it with the maximum rate of $50 \mathrm{Mbps}$. The results show that GaAs/AlGaAs contrast ratio is a key factor to the communication link, which is influenced by drive voltage, incidence angle and modulating speed.
\end{abstract}

Keywords: Free Space Optical Communication; Cat's Eye Effect; Modulating Retroreflector; Multiple Quantum Well.

\section{Introduction}

Compared with microwave communication, free space optical communication (FSO) has many advantages such as high communication rate, secrecy, free electromagnetic applications and so on. Because of the narrow divergence of FSO beam, the acquiring/tracking/pointing (ATP) progress is very hard, and both ends of a FSO link need a laser transmitter, a receiver, a highly accurate ATP system, a control system at least, which makes communication terminal large, heavy and power consumption. When one end of the link cannot accommodate the power consumption or the weight of a FSO terminal, traditional FSO link will not work. To overcome the limit, FSO based on cat's eye modulating retro-reflector (CEMRR) is appropriate. US Naval Research 
Laboratory (NRL)[1] [5], Swedish Defence Research Agency[6] and San Diego Research Center[7] have been doing research on CEMRR. The highest communication speed using GaAs/InAlAs[8] multiple quantum well spatial light modulator (SLM) was 45Mbps reported up to now. Some research on MQW SLM using in CEMRR FSO have been done, including the structure of InGaAs/InAlAs MQW, how MQW SLM works in CEMRR FSO and so on. The already-done research work has proved the possibility and advantages of CEMRR FSO using MQW SLM. In this letter, we introduced the principle of CEMRR FSO using MQW SLM, and analyzed the main influence factors of MQW SLM used in CEMRR. Furthermore, we tested a GaAs/AlGaAs MQW SLM's characteristics when drive voltage and incidence angle varies. At last, we built an experimental CEMRR FSO using GaAs/AlGaAs MQW SLM set with the highest test speed of 50Mbps.

\section{Principle of CEMRR FSO using MQW SLM}

A CEMRR FSO using MQW SLM structure can be shown as Fig 1, which includes 2 parts: active terminal and CEMRR terminal. The active terminal is a traditional FSO terminal, which includes a laser, a receiver, an ATP and a controller. The CEMRR terminal couples a passive cat's eye optical retroreflector with an MQW SLM placed on the focal plane.

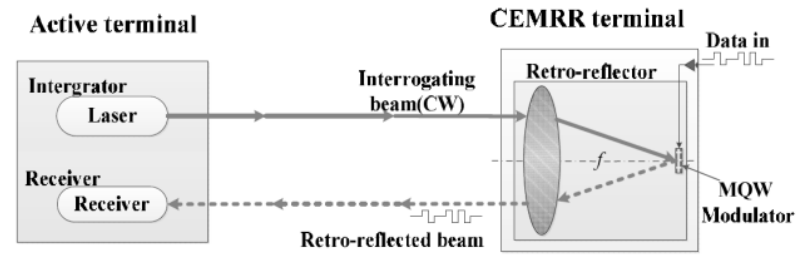

Fig. 1 CEMRR FSO using MQW SLM structure

When the link works, the active terminal transmits a CW laser beam to the CEMRR terminal. Then, the CEMRR terminal receives the incidence beam, modulates the beam according to information to be transmitted by loading the data to driver, and finally retro-reflects the modulated beam to the active terminal to close the link.

Given the interrogating laser power $P_{t}$, laser divergence $\theta_{t}$, interrogator transmissivity $\tau_{t}$, link range $R$, atmospheric transmittance $\tau_{a}$, effective receiving area of MRR terminal $S_{M R R}$, retro-reflector transmissivity $\tau_{M R R}$, CEMRR incidence angle $\varphi$, retro-reflected beam divergence $\theta_{M R R}$, receiver incidence angle $\gamma$, receiver optical transmissivity $\tau_{\text {rec }}$, MQW SLM modulation efficiency $M$, the active terminal finally received power $P_{\text {rec }}$ can be expressed as Eq.(1). The 
link can be closed if the minimum detectable power of receiver detector $P_{\min }$ is smaller than $P_{\text {rec }}$.

$$
P_{r e c}=P_{t} \tau_{t} \tau_{\alpha} \frac{4}{\pi\left(R \theta_{t}\right)^{2}} S_{r e c} \tau_{r e c} \cos \varphi S_{M R R} \tau_{M R R} M \tau_{M R R} \tau_{\alpha} \frac{4}{\pi\left(R \theta_{M R R}\right)^{2}} \cos \gamma
$$

MQW SLM modulation efficiency $M$ can be expressed as Eq. (2), where $e^{-\alpha_{o n}}$ is the absorption product of MQW in its on-state, and $e^{-\alpha_{\text {off }}}$ in its offstate. $C_{M Q W}$ is MQW contrast ratio (CR), which describes the ratio of absorption product when MQW in its on-state and off-state.

$$
\begin{gathered}
M=e^{-\alpha_{o n}}-e^{-\alpha_{o f f}}=e^{-\alpha_{o f f}}\left(C_{M Q W}-1\right) \\
C_{M Q W}=\frac{e^{-\alpha_{o n}}}{e^{-\alpha_{o f f}}}
\end{gathered}
$$

From equations above, in CEMRR FSO, communication link strongly depends on MQW SLM CR. However, for an MQW SLM, the CR is not steady, and it is influenced by many MQW parameters.

\section{Characteristics of GaAs/AIGaAs MQW using in CEMRR FSO}

An MQW SLM is a PIN diode with multiple layers of thin layers of alternating semiconductor alloys in the intrinstic region. The layers consist of a band-gap material, the well, and a higher bandgap material the barrier. MQW devices can be used as SLM based on the quantum-confined stark effect (QCSE), which means when a reverse bias is applied across the MQW the electric field changes the quantum well potential, shifting the exciton feature to the red and reducing the magnitude of the absorption [9]. In this letter, the GaAs/AlGaAs MQW we used is a product of Suzhou Institute of Nano-tech and Nano-bionics, Chinese Academy of Sciences [10][11], its architecture can be shown as Fig 2, an asymmetric Fabry-Perot cavity. The reflectivity spectrum is designed to be shown as Fig 3. Working wavelength is designed around $855 \mathrm{~nm}$, and CR is about 3:1. 

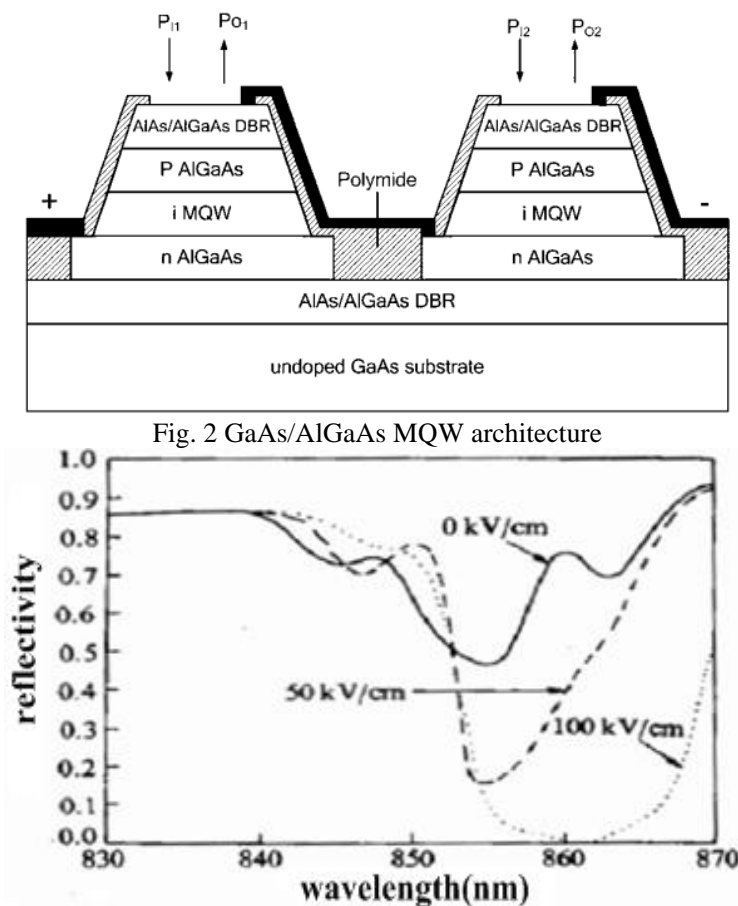

Fig. $3 \mathrm{GaAs} / \mathrm{AlGaAs}$ reflectivity spectrum

The MQW SLM is a $2 \times 2$ array construction, with a size of $4435 \mu \mathrm{m} \times 4435 \mu \mathrm{m}$. Its input impedence is about $50 \mathrm{ohm}$, capacitance about $3 \mathrm{nf}$. The maximum bias voltage is designed to be $6 \mathrm{~V}$. We designed a drive board to supply sufficient current for various driven voltage. The MQW SLM with drive board is shown in Fig. 4. To test how MQW SLM CR varies with drive voltage, we built a test set shown as Fig. 5. The set includs an $850 \mathrm{~nm}$ laser interrogator, a 50/50 beam splitter, a THORLAB FDS100 photodetector, a DG5032 function generator to generate NRZ signal and a Tektronix TDS3032B digital oscilloscope to acquire reflected signal. The MQW SLM is placed on a turntable so as to make the incidence beam perpendicular to MQW SLM surface. 

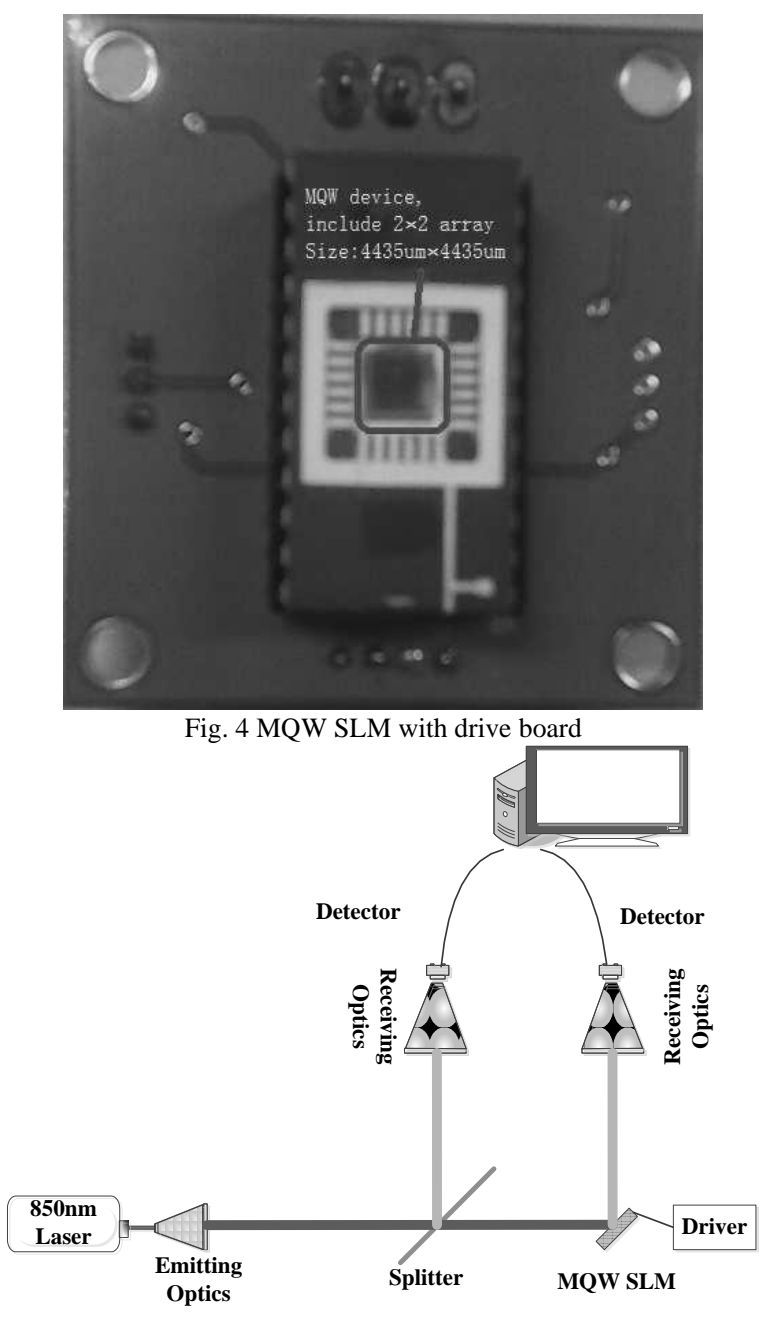

Fig. 5 MQW SLM CR test set

When MQW SLM is driven with a $100 \mathrm{KHz}$ NRZ signal with amplitude varies from $2.8 \mathrm{~V}$ to $3.9 \mathrm{~V}$, the MQW CR v.s. NRZ signal amplitude is shown in Fig. 6. The maximum CR is about 1.24 when NRZ signal's amplitude is $3.9 \mathrm{~V}$, while the minimum CR is about 1.15 when NRZ signal's amplitude is $3.0 \mathrm{~V}$. The MQW SLM CR decreases with drive signal amplitude, and it is much smaller than expected. The MQW SLM can afford a $6 \mathrm{~V}$ bias, while the maximum NRZ 
signal amplitude is $4 \mathrm{~V}$, which made the maximum CR smaller. What's more, MQW SLM is very sensitive to wavelength, the laser we are using is $850 \mathrm{~nm}$, $5 \mathrm{~nm}$ deviating from central wavelength, which is the main factor for MQW SLM CR loss.

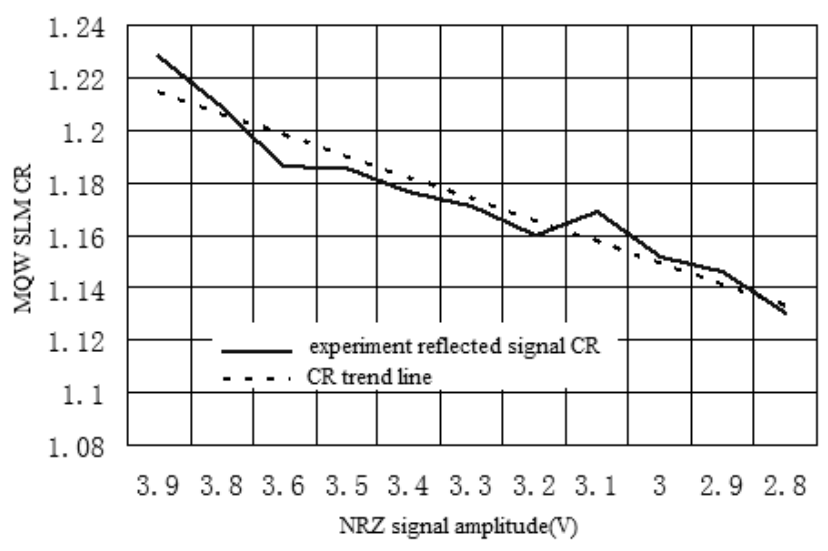

Fig. 6 MQW CR v.s. drive NRZ signal amplitude

When CEMRR FSO works, there will be an angle between incidence beam and MQW SLM surface normal in most cases. When incidence angle varies from -20 degree to 18 degree, MQW CR is shown as Fig. 7. When incidence angle increases, CR decreases. Once the incidence angle is larger than 20 degree, MQW CR is about 1.06 , which is so small that available signal can hardly be recognized.

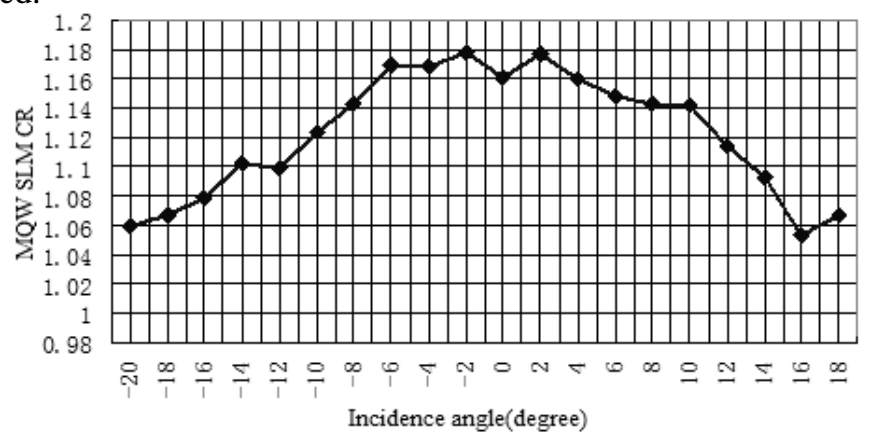

Fig. 7 MQW CR v.s. incidence angle

\section{CEMRR FSO using GaAs/AlGaAs MQW test}

We built a CEMRR FSO experiment set using GaAs/AlGaAs MQW SLM, shown as Fig. 8. It included a laser interrogator, an echo receiver, a beam splitter and a cat's eye target. The cat's eye target couples a thin convex lens with 
GaAs/AlGaAs MQW SLM placed on the focal plane, and its FOV is about 40 degree. When NRZ signal amplitude is $3.9 \mathrm{~V}$, after simple de-noise, retroreflected signal with modulating speed of $100 \mathrm{KHz}, 1 \mathrm{MHz}$ and $50 \mathrm{MHz}$ is shown as (a), (b) and (c) in Fig. 9. The retro-reflected signal amplitude v.s. modulating speed curve is shown as Fig. 10.

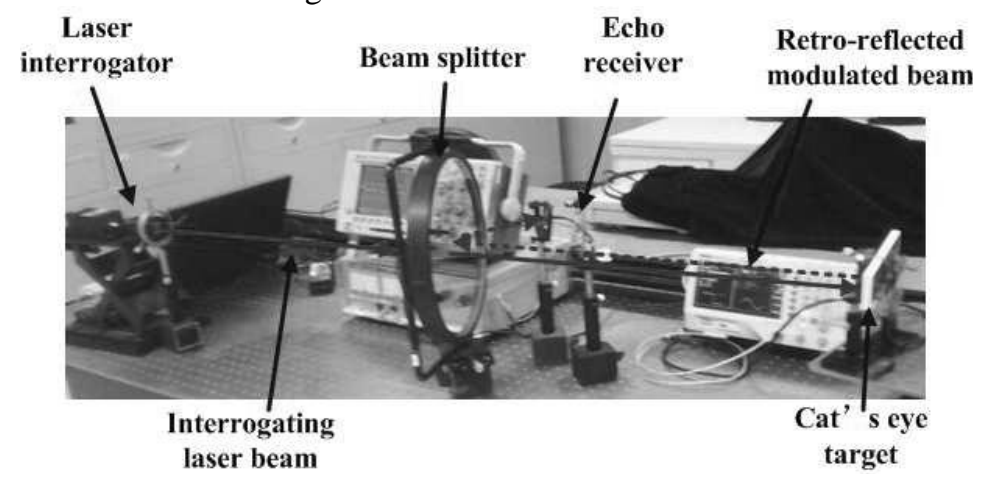

Fig. 8 CEMRR FSO experiment set using GaAs/AlGaAs MQW SLM
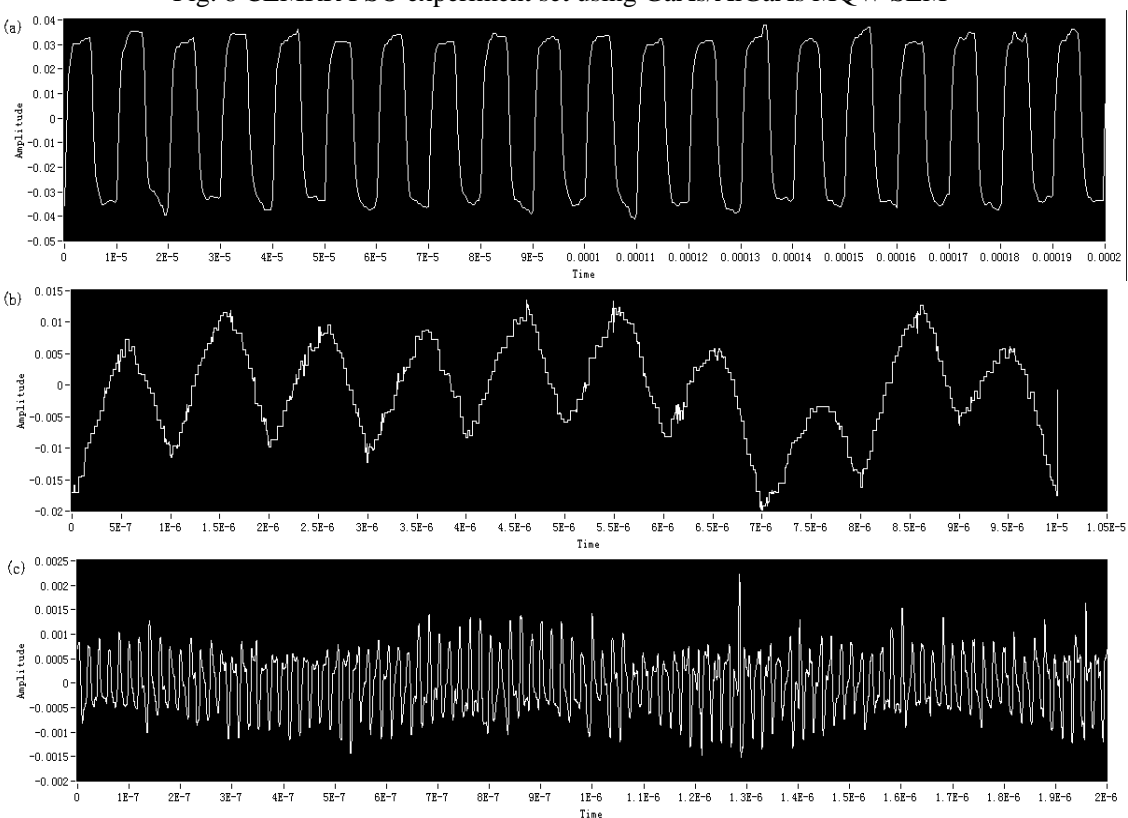

Fig. 9 Retro-reflected signal with modulating speed of (a) $100 \mathrm{KHz}$, (b) $1 \mathrm{MHz}$, and (c) $50 \mathrm{MHz}$ after simple denoise. All the ordinate present the signal amplitude with unit of volt, and abscissa present time with unit of second 


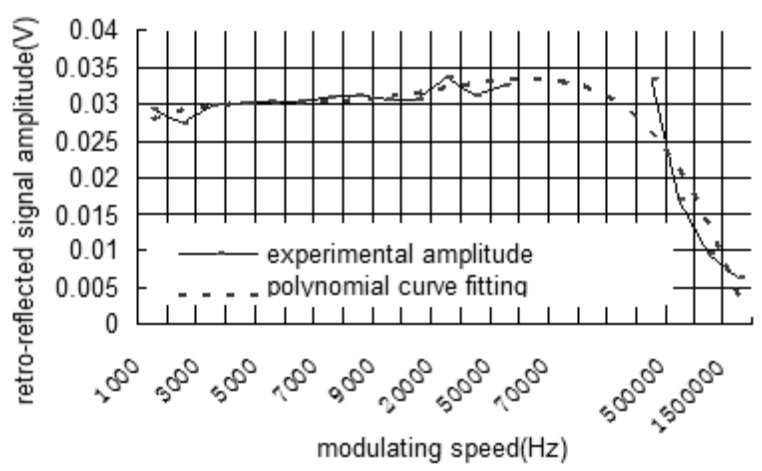

Fig. 10 retro-reflected signal amplitude v.s. modulating speed

The amplitude of retro-reflected signal is stable when modulating speed is relatively lower, and decreases rapidly when modulating speed exceeds certain range. This is because the output impedence (about $50 \mathrm{ohm}$ ) of function generator, input impedence (about $50 \mathrm{ohm}$ ) and capacitance (about 3nf) of MQW LSM build an RC low filter with $3 \mathrm{~dB}$ cutoff frequency of $500 \mathrm{KHz}$. The filter amplitude frequency response curve matches the polynomial curve fitting in Fig. 10, and it attenuates NRZ drive signal amplitude when modulating speed is higher.

\section{Conclusion}

The GaAs/AlGaAs MQW characteristics experiment results clearly show that the GaAs/AlGaAs MQW can be used as an SLM for CEMRR FSO, although its $\mathrm{CR}$ is influenced by drive voltage and incidence angle. When a CEMRR FSO using the GaAs/AlGaAs MQW SLM is built, the maximum data rate is limited by the MQW SLM input impedence and capacitance. Using the MQW SLM in this letter, a CEMRR FSO link can be built when data rate is not too high, which will make rapid FSO link for small platform possible.

\section{Acknowledgments}

This work is supported by the National Natural Science Foundation of China (No.61302183).

\section{References}

1. G. C.Gilbreath, W. S. Rabinovich, R. Mahon, et al. Modulating retroreflector architecture using multiple quantum wells for free space optical communications, in Proc. SPIE 3491, 581-586(1998). 
2. W.S. Rabinovich, G.C. Gilbreath, Peter G. Goetz etc. InGaAs Multiple Quantum Well Modulating Retro-reflector for Free Space Optical Communication, in Proc. SPIE 4489, 190-201(2002).

3. Goetz P.G, Ferraro M.S., Mahon R., et al. Lasercom for high definition video to a moving platform using cat's eye modulating retro-reflectors, in Proc. IEEE Photonics Society Summer Topical Meeting Series, 86-87 (2012).

4. Etai Rosenkrantz, Shlomi Arnon. An innovative modulating retro-reflector for free-space optical communication, in Proc. SPIE 8874, 88740D(2013).

5. Goetz P.G., Rabinovich W.S., Mahon R., et al. Modulating Retro-Reflector Lasercom Systems for Small Unmanned Vehicles, in Proc. IEEE JOURNAL ON SELECTED AREAS IN COMMUNICATIONS, 30(5), 986992(2012).

6. Stéphane Junique, Daniel Ågren, Qin Wang et al. A Modulating RetroReflector for Free-SpaceOptical Communication, PHOTONICS TECHNOLOGY LETTERS, 18(1), 85-87(2006).

7. K.J. Grant, B.A. Clare, W. Martinsen et al. Free Space Optical Transmission of FM Audio/Video Signals Using InGaAs Modulating Retroreflectors, in Proc. 2010 Conference on Optoelectronic and Microelectronic Materials and Devices, (COMMAD 2010), Vol(4), 233-234(2010).

8. W. S. Rabinovich, R. Mahon, P. G. Goetz, et al. 45 Mbps Cat's Eye Modulating Retro-reflector Link Over $7 \mathrm{Km}$, in Proc. SPIE 6304, 63040Q(2006).

9. Miller D A B, Chemla D S, Damen T C, et al. Band-edge electroabsorption in quantum well structures: The Quantum-confined stark effect, Physical Review Letters, 53(22), 2174-2176(1984).

10. Huang Yuyang, Liu H C, Wasilewski Z R, et al. High contrast ratio, high uniformity multiple quantum well spatical light modulators, Journal of Semiconductors, 31(3), 034007(2010). 